\title{
Rapid Progression of Left Ventricular Thrombus with Left Ventricular Dysfunction Detected by Preoperative Transthoracic Echocardiography
}

Satoshi Kimura, MD, Masayoshi Umesue, MD, Sho Matsuyama, MD, and Kanzi Matsui, MD

\begin{abstract}
We report a successfully treated case of rapid progressive left ventricular (LV) thrombus with ischemic cardiomyopathy. Initially, the patient was scheduled to undergo only coronary artery bypass grafting. After two months, preoperative echocardiography revealed a previously undetected ball-like thrombus in the $\mathrm{LV}$ cavity. Surgical revascularization and thrombectomy were performed. No systemic embolism was associated with surgical manipulation during the perioperative period. Repeated preoperative evaluation for the presence of thrombus by transthoracic or transesophageal echocardiography is essential in cases of ischemic cardiomyopathy.
\end{abstract}

Keywords: ischemic cardiomyopathy, left ventricular thrombus, preoperative echocardiography

\section{Introduction}

Left ventricular (LV) thrombus is a common complication of severe LV dysfunction and is associated with the risk of systemic embolism. Echocardiography is a useful tool in the diagnosis of LV thrombus. We report a successfully treated case of rapid progressive LV thrombus detected by echocardiography immediately before the operation.

\section{Case Report}

A 68-year-old man with no obvious history of myocardial infarction was admitted to our institution with shortness of breath on exertion. Transthoracic echocardiography demonstrated diffuse hypokinesis, antero-septal and apical akinesis with impairment of LV function, and an ejection fraction of $28 \%$. Coronary angiogram revealed multiple vessel disease;

Department of Cardiovascular Surgery, Matsuyama Red Cross Hospital, Matsuyama, Ehime, Japan

Received: February 21, 2012; Accepted: June 7, 2012 Corresponding author: Satoshi Kimura, MD, PhD. Department of Cardiovascular Surgery, Matsuyama Red Cross Hospital, 1 Bunkyo-cho, Matsuyama, Ehime 790-8524, Japan

Email: sakimura@heart.med.kyushu-u.ac.jp

(C)2012 The Editorial Committee of Annals of Thoracic and Cardiovascular Surgery. All rights reserved.
90\% stenosis of the right coronary artery, 99\% stenosis of the left anterior descending artery, 90\% stenosis of the diagonal branch, and $90 \%$ stenosis of the third branch. Left ventriculogram demonstrated diffuse severe hypokinesis. No thrombus was identified at that time. He was diagnosed with ischemic cardiomyopathy. The patient was thought to be a good candidate for elective coronary artery bypass grafting (CABG). While awaiting surgery, antiplatelet agents were administered. Two months later, he was re-admitted to our institution for the operation. Preoperative transthoracic echocardiography demonstrated a previously undetected $2.0 \times 1.7 \mathrm{~cm}$ ball-like thrombus in the LV apex (Fig. 1). Therefore, LV thrombectomy via ventriculotomy was scheduled to perform in addition to CABG.

After primary median sternotomy, the left internal thoracic artery and two saphenous vein grafts were harvested for CABG. Cardiopulmonary bypass was established by cannulation of the ascending aorta and right atrium. A vent tube was placed in the left atrium but not the LV to avoid dislodgement of the thrombus. The ascending aorta was clamped, and cardiac arrest was induced by a cardioplegic solution. The LV apex was incised approximately $2 \mathrm{~cm}$ away from and parallel to the left anterior descending artery. A fresh red thrombus attached to the apical muscle was noted. It was meticulously extracted using forceps. After adequate saline irrigation, the ventriculotomy was 


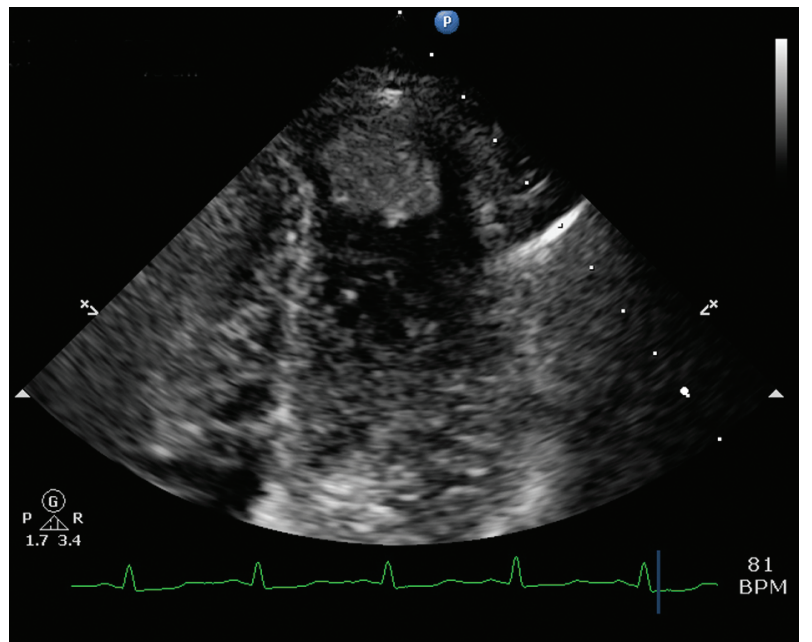

Fig. 1 Preoperative transthoracic echocardiography demonstrated a ball-like thrombus, which developed during the previous 2 months, 1 day before the operation.

directly closed using 3-0 polypropylene sutures with triple felt strips. The proximal ends of the saphenous vein grafts were then anastomosed on to the ascending aorta. After declamping the aorta, the distal ends of the saphenous vein grafts were anastomosed to the third branch and the right coronary artery. The distal end of the left internal thoracic artery was anastomosed to the left anterior descending artery. The patient came off cardiopulmonary bypass with no difficulty. Transesophageal echocardiography revealed no residual thrombus. The postoperative course was uneventful. Oral anticoagulant treatment was administered. The patient was discharged 17 days after surgery with no complications.

\section{Discussion and Conclusion}

LV thrombus is a frequent complication of severe LV dysfunction (such as ischemic cardiomyopathy and dilated cardiomyopathy) and is associated with the risk of systemic embolism. A LV thrombus has been more commonly reported in patients with anterior infarction and poor ejection fraction. ${ }^{1,2)}$ A thrombus develops relatively early after acute myocardial infarction. ${ }^{2)}$ However, the progression rate of a LV thrombus in patients with ischemic cardiomyopathy is unclear. Maslow et al. reported a series of 4 CABG patients in whom unexpected LV thrombus had been detected intraoperatively by transesophageal echocardiography. ${ }^{3)}$ Sharma et al. reported an unrecognized LV thrombus detected by transesophageal echocardiography after decannulation in a patient undergoing coronary revascularization. $\left.{ }^{4}\right)$
In this case, we thought hemoconcentration by diuretics for the prevention of congestive heart failure and diffuse hypokinesis of wall motion contributed to rapid progression of LV thrombus. A previously undetected ball-like thrombus was identified a day before the operation in the LV cavity by transthoracic echocardiography in our patient. Originally, our patient was scheduled to undergo CABG only. If the LV thrombus had not been recognized, an unexpected systemic embolism could have occurred during the perioperative period. When the waiting time for surgery is relative long in such like this case, ischemic cardiomyopathy with severe LV dysfunction, we think it is important to perfome the echocardiography again immediately before the operation from our experience.

Even minimal manipulation of the heart may dislodge or fracture a thrombus. Therefore, cardiac manipulation was performed carefully before aortic clamping to prevent distal embolization and to avoid arrhythmia during surgery. In patients with ischemic cardiomyopathy undergoing coronary revascularization, the possibility of thrombus and the risk of its dislodgement and systemic embolization during the procedure must be considered. Therefore, it is essential to recheck the existence of thrombus by echocardiography just before surgery or at least perioperatively.

\section{Disclosure Statement}

We have no conflict of interests reported in the present paper.

\section{References}

1) Asinger RW, Mikell FL, Elsperger J, et al. Incidence of left-ventricular thrombosis after acute transmural myocardial infarction. Serial evaluation by twodimensional echocardiography. N Engl J Med 1981; 305: 297-302.

2) Visser CA, Kan G, Lie KI, et al. Left ventricular thrombus following acute myocardial infarction: a prospective serial echocardiographic study of 96 patients. Eur Heart J 1983; 4: 333-7.

3) Maslow A, Lowenstein E, Steriti J, et al. Left ventricular thrombi: intraoperative detection by transesophageal echocardiography and recognition of a source of post CABG embolic stroke: a case series. Anesthesiology 1998; 89: 1257-62.

4) Sharma S, Ehsan A, Couper GS, et al. Unrecognized left ventricular thrombus during reoperative coronary artery bypass grafting. Ann Thorac Surg 2004; 78: e79-80. 